\title{
Periodontal Status of Postmenopausal Women with Osteoporosis
}

\author{
${ }^{1}$ Rashmi Paramashivaiah, ${ }^{2}$ Shyam Padmanabhan, ${ }^{3} \mathrm{CD}$ Dwarakanath, ${ }^{4} \mathrm{AV}$ Ramesh \\ ${ }^{1}$ Reader, Department of Periodontology, Krishnadevaraya College of Dental Sciences, Bengaluru, Karnataka, India \\ ${ }^{2}$ Professor, Department of Periodontology, Vydehi Institute of Dental Sciences, Bengaluru, Karnataka, India \\ ${ }^{3}$ Professor and Head, Department of Periodontology, Oxford Dental College and Hospital, Bengaluru, Karnataka, India \\ ${ }^{4}$ Professor, Department of Periodontology, Oxford Dental College and Hospital, Bengaluru, Karnataka, India
}

Correspondence: Rashmi Paramashivaiah, Reader, Department of Periodontology, \#307, First Floor, 6th C Cross, 3rd Main Road, OMBR Layout, Banaswadi, Bengaluru-560033, Karnataka, India, Phone:9900925844, e-mail: rashparams2005@yahoo.co.in

\section{ABSTRACT}

Introduction: Osteoporosis a common metabolic disorder in postmenopausal women, with characteristic loss of bone mass, is associated with periodontitis and tooth loss according to the vast accumulating evidence. This association needs further perusal which was addressed in the following study.

Materials and methods: The study subjects were postmenopausal women (No:104) in the age group of 35 to 60 years. Diagnosis of osteoporosis was by standard tests like clinical, radiographic tests and or bone densitometry. Case history of subjects (i.e. dental, personal, medical and menopausal) was elicited verbally and periodontal examination was carried out. Estrogen (17- $\beta$ estradiol) and calcium assays were measured in 20 of these patients.

Results: All subjects completed their periodontal and radiographic examination. Indices to measure local factors were accompanied by increase in clinical parameters for periodontitis. Alveolar bone loss visible radiographically correlated with clinical indices and clinical attachment loss. Hormonal assays, i.e. serum $17-\beta$. Estradiol and calcium were found to be below normal for examined cases. Majority of patients had periodontitis, half being localized and the remaining generalized.

Conclusion: Mandibular cortex showed osteoporosis in most of the cases. Tooth loss was not an apparent finding. Osteoporosis did not show an association with periodontitis as much as the local environment. Thus, it could be concluded that osteoporosis may not be an important risk factor for periodontitis and oral bone loss.

Keywords: Postmenopausal women, Bone mass, Oral bone loss, Mandibular cortex, Clinical attachment loss, Local factors, Tooth loss, Risk factor.

\section{INTRODUCTION}

Periodontitis characterized by loss of attachment and tooth loss is related to various systemic diseases, like diabetes, respiratory diseases, cardiovascular diseases, adverse pregnancy outcome and osteoporosis.

Osteoporosis is a disease in which the density and quality of bone are reduced, leading to weakness of the skeleton and increased risk of fracture, particularly of the spine, wrist and hip. ${ }^{1}$ Worldwide, lifetime risk for osteoporotic fractures in women is 30 to $50 \%$ and, in men, risk is 15 to $30 \%$. $^{2}$ One out of eight males and one out of three females in India suffers from osteoporosis, making India one of the largest affected countries in the world. ${ }^{3}$ Expert groups peg the number of osteoporosis patients at approximately 26 million (2003 figures) with the numbers projected to increase to 36 million by $2013 .{ }^{4}$

Thus, it becomes imperative to examine the association between osteoporosis and periodontitis as they involve a large strata of population.

\section{MATERIALS AND METHODS}

The Study Population

Postmenopausal women were recruited for the study from the Outpatient Orthopedic Departments of Bowring and Lady
Curzon's Hospital and Santosh Hospital, Bengaluru, private orthopedic clinics and from bone densitometry camps conducted by these hospitals.

\section{Selection of Subjects}

Criteria for inclusion were as follows:

- Postmenopausal women of age group 35 to 60 years

- Presence of at least seven natural teeth in each quadrant

- Diagnosed as osteoporotic by standard tests of diagnosis like clinical with radiographic aids and/or use of bone densitometer.

Exclusion criteria were as follows:

- Patients with diabetes and cancer

- Subjects on hormone replacement therapy for over 6 months

- Patients on long-term steroids and antibiotics over 6 months.

\section{Data Collection}

Upon enrollment of subjects, data regarding the age of attainment of menopause and any problems or surgical intervention associated with it were recorded.

\section{Clinical Examination}

In the hard tissue examination, the number of teeth present, missing teeth and the reasons for loss of teeth were elicited. In 
soft tissue examination, the gingiva was assessed for signs of clinical inflammation.

Debris component of oral hygiene index (Green and Vermillion) ${ }^{5}$ calculus index (NIDR), ${ }^{6}$ gingival index (Loe and Silness) ${ }^{7}$ and bleeding index (Dichotomous) ${ }^{7}$ was recorded.

\section{Periodontal Status}

Parameters such as probing pocket depth, clinical attachment loss, mobility, furcation and pathologic tooth migration were recorded. The periodontal pocket and the clinical attachment were recorded using a William's periodontal probe; furcation was assessed using a Naber's probe.

\section{Osteoporosis Diagnosis}

All 30 of the cases were diagnosed by orthopedecian using clinical examination and radiographic aids. Clinical signs included backache, fracture of the fore arm (Colle's fracture) and fracture on routine activities. Radiographs of the lumbar spine both anteroposterior, lateral and additional radiographs of the fractured sites were obtained.

The remaining 74 of the cases were diagnosed by bone densitometer (Heel Densitometer, Wipro-GE) which measures the density based on ultrasound principle by placing the heel of the foot or pedal region of the patient and comparing the density against the standard bone density of a white German female.

It consists of stiffness index with a T-score and Z-score.

A T-score is as follows:

- 0 to -1 is normal

- $\quad-1$ to -2.5 is osteopenia

- $\quad-2.5$ and below is osteoporosis.

\section{Serum Estrogen and Calcium Assays}

Twenty cases with a T-score below -2.5 were investigated for the estrogen and calcium values.

\section{Radiographic Data}

Panoramic radiographs were obtained for all the 104 participants. Mandibular cortex was examined distally from the mental foramen by oral radiologist and classified into three groups by method of Klemetti ${ }^{8}$ as follows:

- C1-normal cortex with even endosteal margin on both the sides

- C2-moderately eroded cortex with endosteal margin showing semilunar defects (lacunar resorption) or endosteal cortical residues ( 1 to 3 layers) on one or both the sides

- C3-severely eroded cortex with heavy endosteal cortical residues and clearly porous.

The alveolar resorption index was measured by a method described by Wical and Swope. ${ }^{9}$ The measurements were made unilaterally depending on the presence of teeth.

The inferior edge of the mental foramen was traced and a line parallel to the long axis of the mandible and tangential to the inferior border of the mandible was drawn. A line perpendicular to this tangent, intersecting the inferior border of the mental foramen, was constructed.
The following measurements were made along this line using a digital vernier calipers: From the lower border of the mandible to the top of the alveolar crest (mandibular height). The distance from the lower border of the mandible to the inferior edge of the mental foramen (basal height).

Alveolar resorption index = Mandibular height/basal height.

\section{Statistical Methods ${ }^{10,11}$}

Chi-square and Fisher's exact test were used to find the significance association of 17- $\beta$ estradiol with mandibular cortex. Student's t-test (two tailed, independent) was used to find the significance of study parameters between the classes of mandibular cortex. Pearson's correlation coefficient was used to find the relationship of alveolaor resorption index (ARI) with study parameters. Analysis of variance was used to find the association of 17- $\beta$ estradiol with ARI.

\section{RESULTS}

\section{Recruitment}

Over 150 postmenopausal women were interviewed and briefed about this study, but only 104 patients complied to be included in the study for all the examinations.

\section{Osteoporosis Parameters}

\section{General Demography}

The mean age of the participants ranged was $51.99 \pm 6.83$ years. But $36.5 \%$ of patients were in the age group of 56 to 60 years.

\section{Medical Status}

Hypertension was found in a fraction of the population and most of these women were on medication.

\section{Age of Attainment of Menopause}

The mean age of attainment of menopause was $46.47 \pm 4.45$ years. A total of $46.2 \%$ of patients were between 46 and 50 years of age.

\section{PERIODONTAL PARAMETERS}

\section{Debris Index}

The mean debris index score of the participants for the Ramfjord teeth was $1.19 \pm 0.44$. Debris index strongly correlated with mandibular cortex $\mathrm{C} 2$ and alveolar resorption index. A positive correlation was found of debris index and mean clinical attachment loss.

\section{Calculus Index}

The calculus index score of the participants was $1.21 \pm 0.38$. Calculus index correlated with the mandibular cortex C2. Calculus index positively correlated with alveolar resorption index and mean clinical attachment loss. 


\section{Gingival Index}

The mean gingival index score was $0.86 \pm 0.34$. Gingival index strongly correlated with mandibular cortex C2. There was a strong positive correlation of gingival index with mean clinical loss of attachment.

\section{Number of Teeth Present}

A total of $38.3 \%$ of the patients had 26 to 30 teeth remaining, with only $4.7 \%$ of the cases having up to 15 teeth remaining. A total of $19.6 \%$ of the participants had more than 30 remaining teeth of the dentition (Table 1).

\section{Reasons for Loss of Teeth}

A total of $35.6 \%$ of the patients had lost teeth due to caries and $14.45 \%$ of the patients had lost teeth due to periodontitis. About $25.9 \%$ of the cases had combined history of caries and periodontitis for loss of teeth. A total of $23.1 \%$ of the study population had no tooth loss.

\section{Probing Depth}

The mean probing depth for the 104 cases was $3.94 \pm 1.06 \mathrm{~mm}$. There was no correlation of probing depth with alveolar resorption index.

\section{Clinical Attachment Loss}

The mean clinical attachment loss was $5.85 \pm 1.03 \mathrm{~mm}$. There was a strong positive correlation of mean clinical attachment loss with mandibular cortex C2. There was a negative correlation of mean clinical attachment loss with alveolar resorption index (Fig. 1). There was a strong positive correlation of mean clinical attachment loss with debris index, calculus index and gingival index.

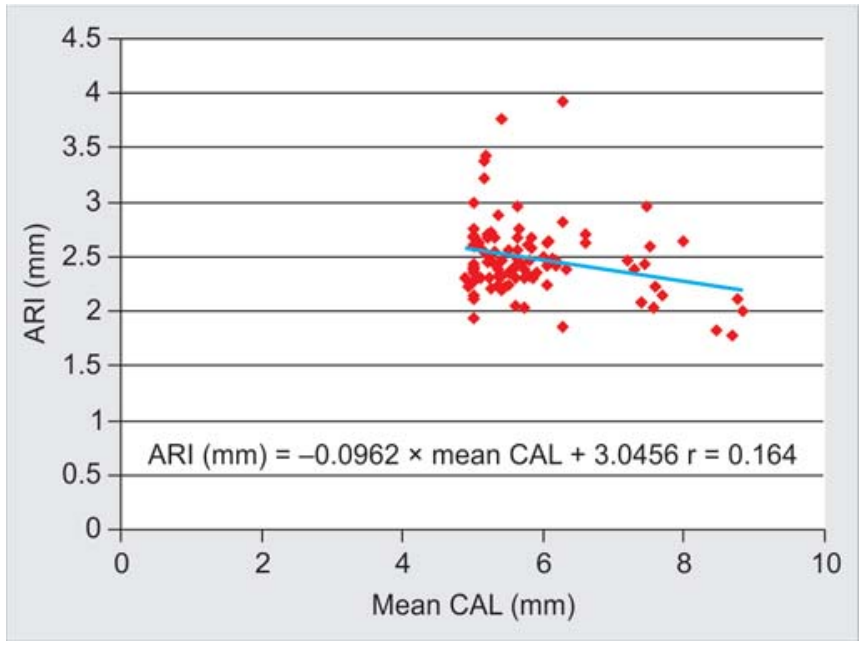

Fig. 1: Scatter plot of mean clinical attachment levels and alveolar resorption index

\section{RADIOGRAPHIC MEASUREMENTS}

\section{Mandibular Cortex}

Mandibular cortex was only in the category of C1 (49.1\%) and C2 (50.9\%) with none of the cases presenting in the C3 category. The mandibular cortex C2 was strongly correlated with age, debris index, gingival index and mean clinical attachment loss.

\section{Alveolar Resorption Index}

The alveolar resorption index was between 2.1 and $2.5 \mathrm{~mm}$ for $55.8 \%$ of the cases and mean alveolar resorption index being $2.49 \pm 0.36 \mathrm{~mm}$ (Table 2). There was no correlation of alveolar resorption index with mandibular cortex. Alveolar resorption index was strongly correlated with debris index and calculus index and moderately correlated with gingival index. There was a negative correlation of alveolar resorption index with mean clinical attachment loss (Fig. 1). There was a positive correlation of alveolar resorption index with 17- $\beta$ estradiol levels (Table 3 ).

\section{BIOCHEMICAL TESTS}

\section{Serum Calcium Levels}

More than $95 \%$ of the 20 subjects had serum calcium levels $<8.5 \mathrm{mg} / \mathrm{dl}$. The remaining $5 \%$ of cases had serum calcium levels $>8.5 \mathrm{mg} / \mathrm{dl}$.

Table 1: Number of teeth present

$\begin{array}{lcc}\begin{array}{l}\text { Number of teeth } \\ \text { present }\end{array} & \begin{array}{c}\text { Number } \\ (n=104)\end{array} \\ \text { Up to } 15 & 5 & 4.7 \\ 16-20 & 14 & 13.1 \\ 21-25 & 23 & 21.5 \\ 26-30 & 41 & 38.3 \\ >30 & 21 & 19.6\end{array}$

Table 2: Alveolar resorption index (ARI) $\mathrm{mm}$

$\begin{array}{lcr}\begin{array}{l}\text { Alveolar resorption } \\ \text { index }(\text { ARI }) \mathrm{mm}\end{array} & \text { Number }(n=104) & \% \\ \leq 2.0 & 5 & 4.8 \\ 2.1-2.50 & 58 & 55.8 \\ 2.51-3.00 & 34 & 32.7 \\ >3.00 & 7 & 6.7 \\ \text { Mean } \pm \text { SD } & 2.49 \pm 0.36 & \end{array}$

Table 3: Association of $17-\beta$ estradiol with alveolar resorption index

$\begin{array}{cc}17-\beta \text { estradiol } \mathrm{pg} / \mathrm{ml} \quad \text { Number of cases } & A R I \\ & \text { mean } \pm S D\end{array}$

$\begin{array}{lcc}\text { Up to } 10 & 13 & 2.37 \pm 0.19 \\ 11-50 & 5 & 2.38 \pm 0.28 \\ >50 & 2 & 2.84 \pm 0.33 \\ \text { Significance } & \mathrm{p}=0.347 & \end{array}$




\section{Serum 17- $\beta$ Estradiol Levels}

Out of the 20 cases assessed, $65 \%$ of the cases had serum $17-\beta$ estradiol levels of up to $10 \mathrm{pg} / \mathrm{ml}, 25 \%$ between 11 and $50 \mathrm{pg} / \mathrm{ml}$ and the remaining $10 \%$ had $>50 \mathrm{pg} / \mathrm{ml}$. There was a moderate positive correlation of 17- $\beta$ estradiol levels with alveolar resorption index (Table 3). Reduced 17- $\beta$ estradiol levels were significantly associated with mandibular cortex C2 (Table 4).

\section{Diagnosis}

A total of $49 \%$ of the cases were diagnosed as chronic generalized gingivitis with localized periodontitis, $48.1 \%$ of the cases being chronic generalized periodontitis and the remaining 2.9\% were chronic generalized gingivitis. Increased incidence of periodontitis both localized and generalized may be associated with reduced levels of 17- $\beta$ estradiol levels.

\section{DISCUSSION}

Osteoporosis and periodontal disease have common risk factors, and both diseases begin to show their effects mainly after the age of 35 years. Considering that periodontal examinations along with radiographic examination are carried out routinely, if any oral signs of osteoporosis can be elicited during these examinations they would prove to be useful for further diagnostic testing.

The study population was homogenous with respect to age, race and socioeconomic status.

The results of the study demonstrated that while periodontitis was prevalent in all but three subjects examined, it was mild to moderate in most of the subjects. Whereas $19.6 \%$ of subjects had all their teeth remaining, $38.3 \%$ of the subjects had more than 25 teeth remaining in the dentition and only $4.7 \%$ had less than 15 teeth. This shows that osteoporosis per se is not a major cause for tooth loss, it was either due to dental caries or periodontitis.

An important finding in the present study was the strong correlation between the local factors and that of the clinical attachment loss and alveolar bone loss. Further, the clinical attachment loss values were strongly associated with alveolar resorption index as well. These findings demonstrated that periodontal status is more determined by local environment than the systemic bone density. These findings are in confirmation with that of study by Elders et al, ${ }^{12}$ Hildebolt et al ${ }^{13}$ and Tezal et al. ${ }^{14}$

Table 4: Association of $17-\beta$ estradiol with mandibular cortex

\begin{tabular}{lcc}
$\begin{array}{l}17-\beta \text { estradiol } \mathrm{pg} / \mathrm{ml} \\
(n=20)\end{array}$ & \multicolumn{2}{c}{ Mandibular cortex } \\
\cline { 2 - 3 } Up to $10(\mathrm{C}=13)$ & $4(20.0)$ & $9(45.0)$ \\
$11-50(\mathrm{n}=5)$ & $5(25.0)$ & - \\
$>50(\mathrm{n}=2)$ & $2(10.0)$ & - \\
Inference & \multicolumn{2}{c}{$\begin{array}{c}\text { Lower estrogen levels associated with } \\
\end{array}$} \\
& the C2 mandibular cortex with \\
& $\mathrm{p}=0.005^{* *}$
\end{tabular}

In the study by Elders et al, Caucasian women between the age group of 46 and 55 years were examined for the relation between systemic bone mass and periodontitis similar to the present study, but the oral bone loss was measured using bitewing radiographs in the molar-premolar junction. The age and number of years since menopause, did not seem to have a bearing on the number of missing teeth, mean probing depth or the occurrence of bleeding on probing which was the case in the present study. Even the correlation between the alveolar bone loss and bone mineral density could not be observed. ${ }^{12}$

The association between bone mineral density and periodontitis was studied in older patients where in addition to periodontitis, smoking history, body mass index, missing teeth and dietary calcium were recorded. Bone mineral density (BMD) was measured with dual energy X-ray absorbsiometry (DEXA) and periodontal parameters were measured with advanced diagnostic aids. ${ }^{13}$ Here, there was no correlation between systemic bone mineral density and clinical attachment loss similar to the present study.

According to the study by Tezal et al, BMD was found to be related to interproximal alveolar bone loss which was determined using intraoral-periapical and bite-wing radiographs. But clinical attachment loss was found to have no relation with the systemic bone similar to the present study. In contrast to the present study, there was significant correlation between skeletal BMD and interproximal alveolar bone loss. ${ }^{14}$

A large sample of osteoporotic subjects belonging to diverse racial background were studied by Persson et al for assessing the relation between BMD and oral bone loss. The results of the study demonstrated that osteoporosis and periodontitis were strongly associated unlike the present study. But this study used only radiographic assessment of periodontitis without clinical parameters, which may have led to these results. ${ }^{15}$

Studies by national health and nutrition survey have shown that maintaining calcium levels at old age by increased dietary intake, has a beneficial role in preventing periodontitis. ${ }^{16}$ Keeping these data in view levels of serum calcium were estimated in every fifth patient in the present study. Around $95 \%$ of the subjects had calcium levels ranging between 7.32 and $8.58 \mathrm{mg} / \mathrm{dl}$. Although these levels were marginally less than normal level of $8.5 \mathrm{mg} / \mathrm{dl}$ no conclusive correlation could be drawn between the serum calcium levels and periodontal status.

Similarly, serum 17- $\beta$ estradiol levels have been correlated with alveolar crestal height by Hildebolt et al, ${ }^{17}$ where they have observed a strong correlation between the two. The normal levels of serum 17- $\beta$ estradiol, in postmenopausal females, are usually between 30 and $40 \mathrm{pg} / \mathrm{ml} .{ }^{18}$ It is significant that 18 of the 20 cases examined for this substance had levels lower than $30 \mathrm{pg} / \mathrm{ml}$ who might have required hormone replacement therapy. However, the periodontal status did not greatly vary in these patients, although statistically there was more attachment loss as well as bone loss in these patients. The poor size of the sample could perhaps explain this discrepancy.

The present study had a main limitation of lack of more sophisticated diagnostic aids like DEXA or subtraction 
radiography which are more precise for alveolar bone loss. A larger sample size would have probably given more information regarding the association between these two entities.

But to find an association between these two diseases which are affected by so many variables it is essential to have a welldesigned, longitudinal prospective clinical trial wherein having matched controls would probably aid in obtaining a clearer picture.

\section{ACKNOWLEDGMENTS}

I would like to thank my family for their unconditional support during the entire study. I would like to thank the staff of Department of Periodontics of Oxford Dental College. A special thanks to Dr GV Prashanth and Dr Savita AN who were a constant motivational power during the entire project. Finally, I would like to thank the almighty God for making everything posssible.

\section{REFERENCES}

1. International Osteoporosis Foundation (IOF). http://www. iofbonehealth. org/patients-public/about-osteoporosis. html

2. Randell A, Sambrook PN, Nguyen TV, et al. Direct clinical and welfare costs of osteoporotic fractures in elderly men and women. Osteoporos Int 1995;5:427.

3. http://www. outlookindia. com 2004.

4. Osteoporosis Society of India. Action plan osteoporosis: Consensus statement of an expert group. New Delhi 2003.

5. John C, Greene. The oral hygiene index: Development and uses. American Journal of Public Health 1960;7:53-63.

6. Miller AJ, Brunelle JA, Carlos JP. Oral health of United States adults. NIDR publication no (NIH) 1987;87-2868. Bethesda, MD, US, Public Health Service, US Department of Health and Human Services.

7. Loe H, Silness J. Periodontal disease in pregnancy. Acta Odontol Scand 1963;21:532-51.
8. Klemetti E, Kolmakov S, Kroger H. Pantomography in assessment of the the osteoporosis risk group. Scand J Dent Res 1994;102:68-72.

9. Wical KE, Swoope CC. Studies of residual ridge resorption (Part I). Use of panoramic radiographs for the evaluation and classification of mandibular resorption. J Prosthet Dent 1974;32:7-12.

10. Bernard Rosner. Fundamentals of Biostatistics (5th ed), Duxbury 2000.

11. Reddy Venkataswamy M. Statistics for mental health care research. NIMHANS publication, India 2002.

12. Elders PJM, Habets LLMH, Netelenbos JC, Van der Linden LWJ, Van der Stelt PF. The relation between periodontitis and systemic bone mass in women between 46 and 55 years of age. J Clin Periodontol 1992;19:492-96.

13. Hildebolt CF, Pilgram TK, Dotson M, Yokoyama-Crothers N, Muckerman J, Hauser J, et al. Attachment loss with postmenopausal age and smoking. J Periodont Res 1997;32: 619-25.

14. Mine Tezal, Jean Wactawski-Wende, Grossi Sara G, HO Alex W, Dunford Robert, Genco Robert J. The relationship between bone mineral density and periodontitis in postmenopausal women. J Periodontol 2000;71:1492-98.

15. Persson RE, Hollender LG, Powell LV, Mac Entee MI, Wyatt CCL, Kiyak HA, et al. Assessment of periodontal conditions and systemic disease in older subjects I. Focus on osteoporosis. J Clin Periodontol 2002;29:796-802.

16. Nishida M, Grossi SG, Dunford RG, Ho AW, Trevisan M, Genco RJ. Calcium and the risk for periodontal disease. J Periodontol 2000;7:1057-66.

17. Hildebolt CH, Pilgram TK, Yokoyama-Crothers Naoko, Vannier Michael W, Roberto Civitelli. The pattern of alveolar crest change in healthy postmenopausal women after 3 years of hormone/estrogen replacement therapy. J Periodontol 2002;73:1279-84.

18. Reinhardt Richard A, Payne Jeffrey B, Maze Connie A, Patil Kashinath D, et al. Influence of estrogen and osteopenia/ osteoporosis on clinical periodontitis in postmenopausal women. J Periodontol 1999;70:823-28. 